\title{
Simulation of attosecond streaking of electrons emitted from a tungsten surface
}

\author{
C. Lemell, ${ }^{1}$ B. Solleder,${ }^{1}$ K. Tőkési, ${ }^{2}$ and J. Burgdörfer ${ }^{1}$ \\ ${ }^{1}$ Institute for Theoretical Physics, Vienna University of Technology, \\ Wiedner Hauptstraße 8-10, A-1040 Vienna, Austria \\ ${ }^{2}$ Institute of Nuclear Research of the Hungarian Academy of Sciences, \\ (ATOMKI), H-4001 Debrecen, P.O.Box 51, Hungary
}

(Dated: October 27, 2018)

\begin{abstract}
First time-resolved photoemission experiments employing attosecond streaking of electrons emitted by an XUV pump pulse and probed by a few-cycle NIR pulse found a time delay of about 100 attoseconds between photoelectrons from the conduction band and those from the $4 \mathrm{f}$ core level of tungsten. We present a microscopic simulation of the emission time and energy spectra employing a classical transport theory. Emission spectra and streaking images are well reproduced. Different contributions to the delayed emission of core electrons are identified: larger emission depth, slowing down by inelastic scattering processes, and possibly, energy dependent deviations from the free-electron dispersion. We find delay times near the lower bound of the experimental data.
\end{abstract}

PACS numbers: $79.60 . \mathrm{Bm}, 72.10 .-\mathrm{d}, 78.47 . J-$ 


\section{INTRODUCTION}

Photoemission from solid surfaces employing photon energies ranging from the extended ultraviolet $(\mathrm{XUV})$ to $\mathrm{X}$-ray energies is a well established diagnostics tool to explore the electronic band structure [1], the crystal structure [2], and its chemical composition [3]. High-resolution angle resolved photoemission can provide detailed information on band gap and conical intersections, e.g. in single-layer graphene [4]. EXAFS allows probes of the local environment by spectral fluctuations due to interfering paths [5]. Until recently, virtually all of these techniques were based on the extraction of spectral information only, i.e. on the energy distribution $P\left(E_{e}\right)$ for a given initial photon energy $E_{\gamma}$. The use of pulsed sources on the femtosecond scale has recently given access to dynamical processes, e.g. the formation of image states [6] .

The introduction of the attosecond streaking technique [7], originally developed for gasphase photoionization, to photoemission from solid surfaces has opened up a new perspective: observation of electronic motion in condensed matter and near surfaces in real time. The first proof of principle experiment revealed that XUV photoemission from a tungsten surface features an intriguing time structure with the conduction band electrons coming first while electrons from core levels, in the present case mostly $4 \mathrm{f}$ and 5p, are delayed by about 100 attoseconds (110 \pm 70 as) [8].

Analyzing the energy-time spectrum and identifying possile sources of the delay poses a considerable challenge to a microscopic simulation: apart from the intrinsic difficulties in accounting for the many-body response on a sub-femtosecond scale, the near infrared (NIR) probe with intensities of $I=2 \cdot 10^{12} \mathrm{~W} / \mathrm{cm}^{2}$ is sufficiently strong as to actively modify the electronic response and emission process. Deviations from a field-free emission scenario are therefore to be expected. We present in the following a simulation of the attosecond streaking of tungsten employing a classical transport theory (CTT, [9]). It allows to account for elastic and inelastic scattering processes as well as modifications of emission energy and angular distributions due to the presence of the streaking field. We find, overall, good agreement with the attosecond-streaking emision spectrum. The time spectrum shows, indeed, delayed photoemission with a delay time $\tau \approx 40$ as being at the lower bound of the experimental value. We present a detailed breakdown of different processes taken into account influencing the time spectrum and discuss possible additional contributions. 


\section{SIMULATION}

Before presenting the key ingredients entering the theoretical treatment, we briefly review the experiment underlying the scenario of the present simulation [8].

Two collinear linearly polarized laser pulses with the polarization direction in the plane of incidence were directed on a W(110) surface under a grazing angle of incidence (Fig. 1). The two pulses were an XUV pulse at $91 \mathrm{eV}$ with a full width at half maximum

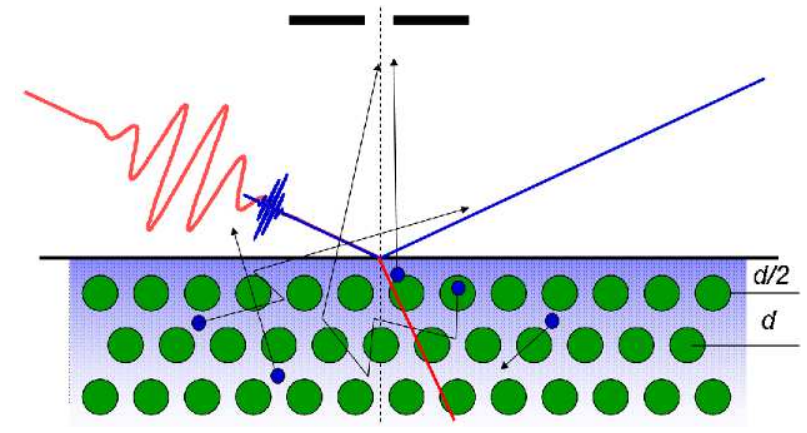

FIG. 1: (Color online) Schematic view of experimental setup: A 300 as XUV pulse hits the surface under a gracing angle of incidence $\theta_{i n} \simeq 75.5^{\circ}$ ionizing target atoms. Excited electrons propagate in the field of a 10 fs NIR pulse and are detected by a TOF spectrometer mounted perpendicular to the surface with an acceptance cone of half-width $\Delta \theta=5^{\circ}$.

(FWHM) of $\sim 6 \mathrm{eV}$ and a pulse duration $\tau_{X U V} \simeq 300$ as and a NIR pulse at $702 \mathrm{~nm}$ with $\tau_{N I R} \simeq 10 \mathrm{fs}$. The angle of incidence was chosen to be at the Brewster angle for the probe pulse in order to avoid the influence of the deflected pulse on electrons emitted from the surface $\left(\theta_{i n}=\arctan \left(n_{N I R}\right) \simeq 75.5^{\circ} ; n_{N I R}=3.85, k_{N I R}=2.86,[10]\right.$; dielectric function $\left.\varepsilon=\varepsilon_{1}+i \varepsilon_{2}=\left(n^{2}-k^{2}\right)+2 i n k\right)$. This implies for the XUV pulse $\left(n_{X U V}=0.93, k_{X U V}=0.04\right.$; [10]) total reflection at the surface and excitation of photoelectrons by an evanescent wave. Electrons excited by the pump pulse propagate in the field of the probe pulse modulating their energy as a function of their emission time ("streaking", [7]). Photoelectrons escaping the target surface were detected by a time-of-flight spectrometer with the detection direction normal to the surface. 40 streaking spectra were recorded with relative delays between pump and probe pulses ranging from -6 fs to +5 fs with respect to the maxima of the envelope functions of XUV and NIR pulses.

On top of a strong background signal originating from above-threshold ionization (ATI) 
two prominent features were observed in each of the streaking spectra: a lower energy peak around $55 \mathrm{eV}$ and a higher energy peak at $85 \mathrm{eV}$. The former was attributed to electrons excited from $4 \mathrm{f}$ core states of tungsten, the latter to electrons from the target conduction band (consisting of $5 \mathrm{~d}$ and 6 s states).

The incident XUV spectrum has been determined by measuring the kinetic energy of electrons emitted from a Ne gas target (Fig. 2). Two main peaks from emission of $2 \mathrm{~s}$ and $2 \mathrm{p}$ electrons with binding energies of $48.5 \mathrm{eV}$ and $21.65 \mathrm{eV}$, respectively, can be distinguished. We have fitted the peak at $70 \mathrm{eV}$ by a Gaussian function (solid line) from which the XUV photon energy of about $91 \mathrm{eV}$ and the approximate FWHM of $6 \mathrm{eV}$ were derived.

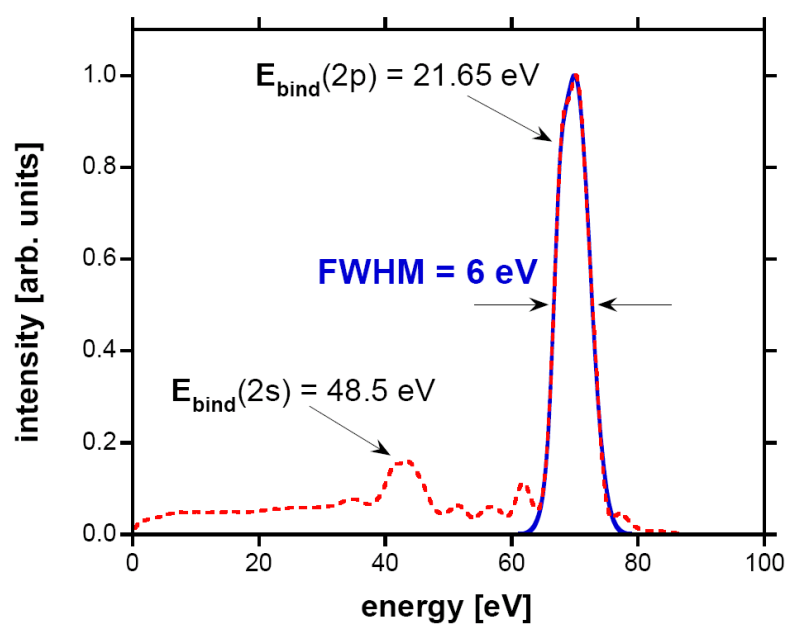

FIG. 2: (Color online) Kinetic energy of electrons emitted from Ne irradiated by the XUV pulse used in the present surface experiment (red dotted line); spectral distribution used in simulation (blue solid line). From the binding energy of the $\mathrm{Ne}(2 \mathrm{p})$ level the XUV photon energy of $\sim 91 \mathrm{eV}$ was determined.

The simulation involves the solution of the Langevin equation of motion,

$$
\dot{\vec{p}}=-\vec{F}_{N I R}(\vec{r}, t)+\vec{F}_{\text {stoc }}(t)+\vec{F}_{\text {surf }}(\vec{r})
$$

for an ensemble of a large number (typically $10^{7}$ ) of initial conditions representing the primary photoionization electrons. $\vec{F}_{\text {stoc }}(t)$ allows for both elastic and inelastic scattering of the liberated electron with tungsten cores as well as conduction electrons

$$
\vec{F}_{s t o c}(t)=\sum_{i} \Delta \vec{p}_{i} \delta\left(t-t_{i}\right)
$$


where $\Delta \vec{p}_{i}$ is the random collisional momentum transfer as deduced from differential scattering cross sections. $t_{i}$ are the random collision times determined from a Poisson distribution with a mean value given by the mean free path (MFP) or, equivalently, the mean flight time. The key point of this method is that the stochastic sequence $\left(\Delta \vec{p}_{i}, t_{i}\right)$, determined either from quantum calculations or independent experimental data, allows quantum scattering information including diffraction to be included in an otherwise classical calculation (Eq. 1, [9]). In between collisions, free electrons with an effective mass (the value of which is discussed below) propagate within the penetration depth of the time-dependent NIR electric field. Near the surface, the electrons must overcome the effective surface potential, the asymptotic part of which is the dynamical image potential [11]. This gives rise to the additional force $\vec{F}_{\text {surf }}(\vec{r})$ in Eq. 1.

As collisions can result in secondary electron emission, the simulation does not preserve the number of particles but allows "en route" for the generation of additional trajectories thereby simulating the collision cascade. However, slow secondary electrons generated outside the energy window of interest are omitted in the following.

\section{A. Primary photoemission as initial condition}

The ensemble of initial conditions represents photoemission of tungsten electrons by a 91 eV XUV pulse which originate from 5 atomic levels: 6s, 5d, 4f, 5p, and 5s. Photoelectrons from the latter $\left(E_{\text {bind }}(5 s)=75.6 \mathrm{eV}\right)$ are submerged in the ATI background and are therefore omitted in our simulation. Electrons excited from the $4 \mathrm{f}$ and $5 \mathrm{p}$ levels (referred to in the following as core levels) give rise to a peak near $55 \mathrm{eV}$ kinetic energy. The binding energies of the $4 \mathrm{f}_{5 / 2}$ and $4 \mathrm{f}_{7 / 2}$ states are $33.5 \mathrm{eV}$ and $31.4 \mathrm{eV}$, respectively. The branching ratio for photon energies around $100 \mathrm{eV}$ of $R_{I}=I_{7 / 2} / I_{5 / 2}=1.56$ was taken from experimental photoemission data [12]. With a binding energy of $36.8 \mathrm{eV}$ the energy of excited 5 $\mathrm{p}_{3 / 2}$ electrons overlaps with the distributions of $4 \mathrm{f}$ electrons. Due to the smaller photoexcitation cross section its relative importance is about a factor 3 smaller than the combined $4 \mathrm{f}$ levels [13]. Excitation of the $4 \mathrm{f}$ and $5 p$ levels with the XUV pump pulse taking the 4f-branching ratio into account leads to a slightly asymmetric energy distribution of primary electrons around $58 \mathrm{eV}$. For brevity, we refer to this overlapping distribution in the following as the core level. 
The $5 \mathrm{~d}$ (4 electrons per atom) and 6s (2 electrons per atom) levels of tungsten form the conduction band. Its density of states (DOS) was taken from [14] (Fig. 3). The Fermi energy is $9.75 \mathrm{eV}$ while the workfunction of $\mathrm{W}(110)$ is taken to be $5.25 \mathrm{eV}$ [15]. We split the DOS in two components: a free-electron gas like subband containing the 6 s electrons $(\operatorname{DOS} \propto \sqrt{E}$; area below red line) and a more localized component representing the 5 d electrons. A similar decomposition of the DOS with a slightly higher value for the Fermi energy has already been proposed earlier by Mattheiss [16]. He suggested the bottom of the 6 s band to lie below the bottom of the 5 d band, as shown in Fig. 3. Density-functional theory calculations using the

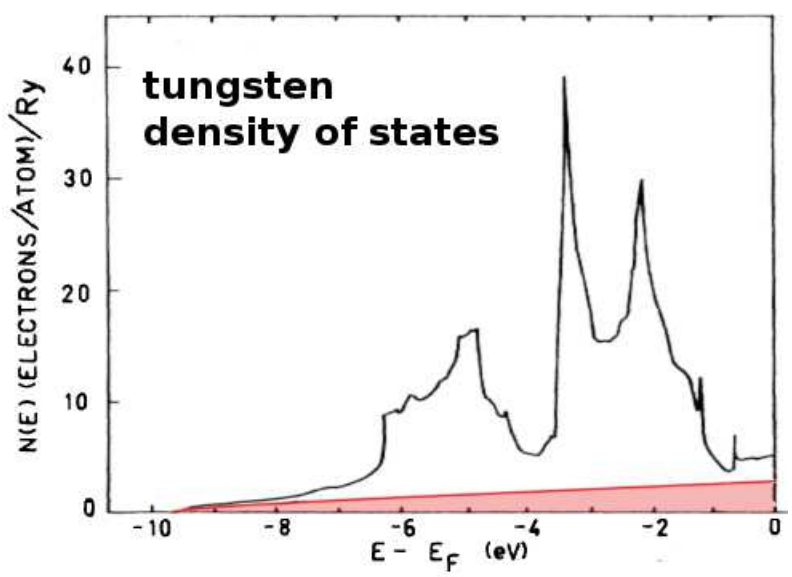

FIG. 3: (Color online) Occupied part of the density of states of tungsten [14]. States below the red solid line (shaded area) originate from the 6 s band, states above the line from the (more localized) 5 d level.

ABINIT package [17] with pseudopotentials for the tungsten cores [18] give a Fermi energy of $E_{F}=9.6 \mathrm{eV}$ in good agreement with [14] and [16].

We note that in the spectral region of interest an additional emission channel may contribute: Auger decay from the conduction band filling a 5s hole created by photoionization (binding energy of the $5 \mathrm{~s}$ level $\sim 75.6 \mathrm{eV}$ ). The expected energy range for this Auger emission would be 45 to $65 \mathrm{eV}$. We expect, however, that the refilling of $5 \mathrm{~s}$ holes will proceed predominantly via the $5 \mathrm{~s} 5 \mathrm{p}^{2}$ decay channel. This is also supported by XPS spectra of the W 4f region in which no signatures of Auger electron emission have been seen (e.g. [19]). Therefore, we do not include this channel in our simulation.

We set the emission strength (dipole oscillator strength) for each level to be constant within the spectral width of the XUV pulse. For the relative intensity ratios between differ- 
ent levels, we have explored two different options. One uses the calculated photoionization cross sections for atomic tungsten [13]. Accordingly, the photoionization cross section for the $4 \mathrm{f}$ subshell of atomic tungsten is by about a factor two larger than for the $5 \mathrm{~d}$ level and by more than an order of magnitude larger than for 6 s electrons. Using this input we find a spectral distribution (in absence of any streaking field) in strong disagreement with experimental data. Experimental photoemission cross sections from the conduction band appear to be considerably larger than estimated from atomic cross sections. Alternatively, we have adjusted the strength of the conduction band peak relative to the core peak in order to reproduce a photoelectron spectrum after excitation by an XUV pulse (in the absence of the NIR field - thick red line in Fig. 4). As the experimental spectrum includes the modification

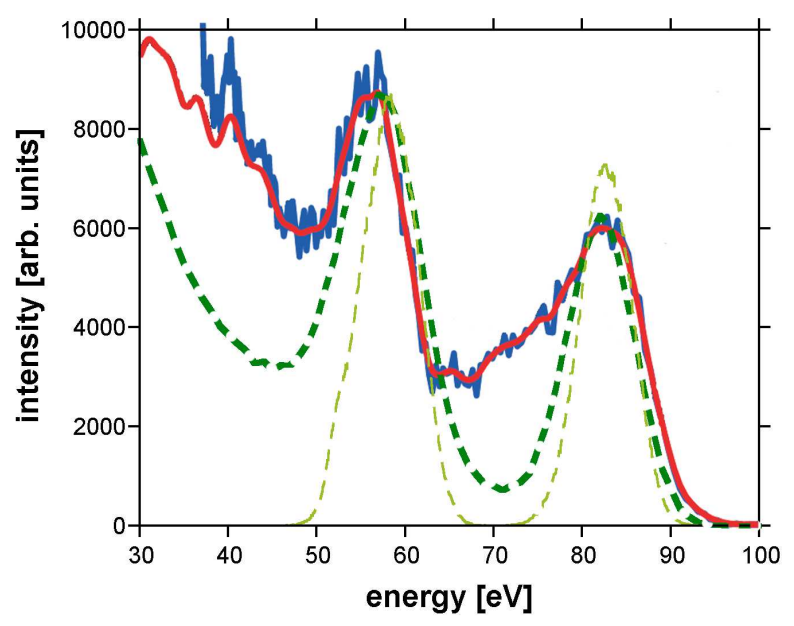

FIG. 4: (Color online) Energy spectra of electrons emitted by an XUV laser pulse in absence of the NIR streaking pulse from a tungsten surface. Experimental data with the ATI background subtracted are indicated by thick solid lines (blue - raw spectrum, red - smoothed raw spectrum), results of the CTT simulation by the thick dashed line. The thin dashed line represents the excitation spectrum as represented by the initial conditions.

of the primary spectrum due to multiple scattering during transport until electrons cross the metal-vacuum boundary, a proper comparison must account for these processes as well (for details see below). Including transport and broadening of the emission spectrum to simulate the detector resolution (Gaussian distribution, $\sigma=2.5 \mathrm{eV}$ ) the simulated photoemission spectrum (thick dashed line) fits the experimental data (raw data with the ATI background subtracted) rather well. A notable exception is the low-energy shoulder of the $80 \mathrm{eV}$ con- 
duction band peak the origin of which is presently not known. We note parenthetically that a very recent measurement shows a much reduced shoulder [20].

The angular distribution of primary photoelectrons excited by linearly polarized photon beams is given in dipole approximation by

$$
\frac{d \sigma_{n \ell}(\omega)}{d \Omega}=\frac{\sigma_{n \ell}(\omega)}{4 \pi}\left(1+\beta(\omega) P_{2}(\cos \theta)\right)=\frac{\sigma_{n \ell}(\omega)}{4 \pi}\left(1+\frac{\beta(\omega)}{2}\left(3 \cos ^{2} \theta-1\right)\right)
$$

where $\sigma_{n \ell}$ is the total photoionization cross section from the $n \ell$ subshell, $P_{2}$ is the second order Legendre polynomial, and $\beta(\omega)$ is the energy-dependent asymmetry parameter. $\beta=0$ describes isotropic emission while a value of $\beta=2$ gives a pure cosine distribution. Calculated values for beta for the considered levels vary from $\beta=0.7$ (5p) to $\beta=2$ for s-levels [13]. Other sources, however, suggest different values and experimental data are, to our knowledge, missing. We have therefore performed simulations for the limiting cases of $\beta=0$ and $\beta=2$ but have found no significant influence of the specific choice on our final results. As an evanescent wave propagates parallel to the surface, its polarization vector is perpendicular to the surface $(\theta=0)$. All results presented in Sec. III were calculated using $\beta=2$ (cosine distributions along surface normal) for all levels.

For the modification of the primary photoionization spectrum by multiple scattering and, in turn, for analyzing the time structure underlying the emission spectrum, the spatial distribution of the primary excitation, i.e. the depth profile of the source is of crucial importance. Starting positions of electron trajectories were distributed over the 20 topmost layers of a $\mathrm{W}(110)$ crystal (lattice parameter $a_{W}=3.16 \AA$, layer spacing in $\langle 110\rangle$ direction $d=2.24 \AA$ ). For the free-electron like $6 \mathrm{~s}$ component of the conduction band (Fig. 3) we use the jellium approximation, i.e. constant density within the target material starting half a layer spacing above the topmost atomic layer. For the 5d subband of the conduction band as well as for the core electrons localized source distributions were chosen from Gaussian distributions with a FWHM of half a layer distance centered at crystal layer positions in agreement with our ab-initio calculations.

The probability of excitation was assumed to be proprtional to the attenuated intensity of the XUV laser light entering the crystal. For $\theta_{i n}=75.44^{\circ}$ and $n_{X U V}=0.93$ total reflection conditions are fulfilled and only an evanescent wave enters the target. The decay length is about $\delta \approx 0.55 \cdot \lambda \approx 75.6 \AA$ or, equivalently, more than 30 layer spacings. The depth dependent reduction in ionization probability has been taken into account, the first five 
layers of which are shown in Fig. 5 ,

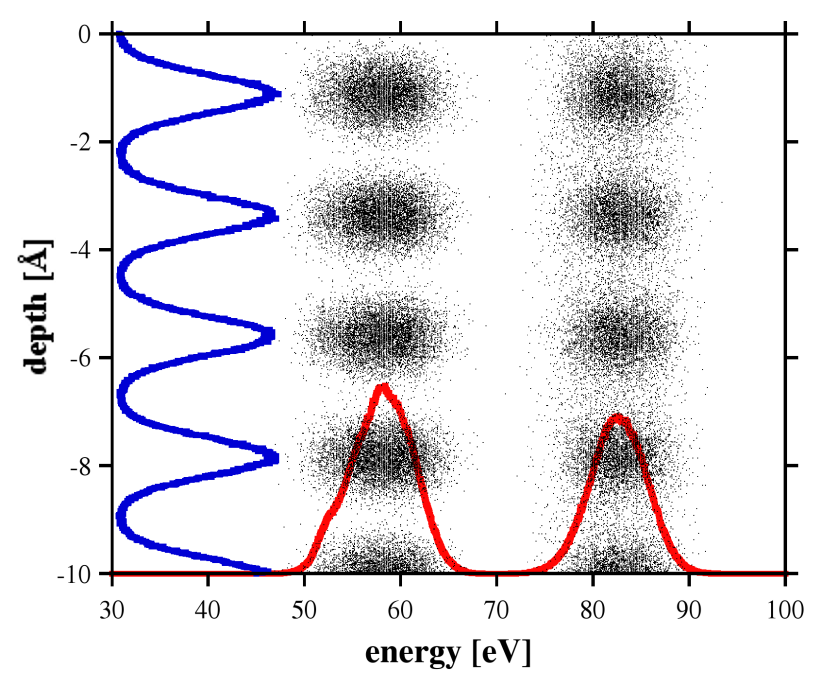

FIG. 5: (Color online) Depth-dependent spectral distribution of primary photoelectrons excited by the XUV pulse with mean energy $91 \mathrm{eV}$, shown for the five topmost crystal layers. Projections onto the energy axis and the depth axis indicate the spectral and depth profiles.

\section{Electron transport}

Electron transport within the target material is calculated using a classical transport simulation described in detail in [21]. In brief, an electron released at its starting point with initial kinetic energy $E_{k i n}$ is subject to elastic and inelastic scattering processes as well as to deflection of its trajectory due to the influence of the electric field of the probe laser pulse in the target (Eq. 1). The latter is refracted and damped by the target material. For $n_{N I R}=3.85$ and $k_{N I R}=2.86$ at $\lambda=702 \mathrm{~nm}$ we find a propagation angle of $\theta \approx 14.5^{\circ}$ with respect to the surface normal. The NIR intensity after a propagation length $l$ is described by the Beer-Lambert-Law

$$
I(l)=I_{0} \cdot \exp \left\{-\frac{4 \pi k(\lambda)}{\lambda} \cdot l\right\}=I_{0} \cdot \exp (-\alpha l)
$$

with the damping constant $\alpha=0.005 \AA^{-1}$ corresponding to a penetration depth of about 85 layers into the target.

Elastic scattering cross sections have been calculated with the ELSEPA package [22] using a muffin-tin potential for the crystal atoms. From the energy-dependent total cross section 
and the density of tungsten atoms the elastic mean free path (EMFP) is derived (Fig. [5). The scattering angle in an elastic scattering event is determined by the energy-dependent differential cross sections.

The inelastic scattering mean free path (IMFP) is derived from the momentum and energy dependent dielectric constant of the material $\varepsilon(q, \omega)$ as [23]

$$
\frac{d^{2} \lambda_{\text {inel }}^{-1}}{d q d \omega}=\frac{1}{\pi E q} \Im\left\{-\frac{1}{\varepsilon(q, \omega)}\right\} \Theta\left[\omega_{m}(q)-\omega\right]
$$

while the angular distribution of inelastically scattered electrons follows from [24]

$$
\frac{d \lambda_{\text {inel }}^{-1}}{d \Omega}=\frac{1}{\pi^{2}} \int \frac{d \omega}{q^{2}} \sqrt{1-\frac{\omega}{E}} \Im\left\{-\frac{1}{\varepsilon(q, \omega)}\right\} \Theta\left[E-E_{f}-\omega\right] .
$$

In Eqs. 5 and 6 E is the instantaneous energy of the electron measured relative to the bottom of the conduction band while $\omega$ and $q$ are the collisional energy and momentum transfers, respectively. The $\Theta$ (step) functions impose the constraints of energy and momentum conservation in the scattering event. $\varepsilon(q, \omega)$ is constructed from an extrapolation of the optical data $[\varepsilon(q=0, \omega)]$ for tungsten [10] to the $q-\omega$ plane (e.g., [25, 26]). The resulting inelastic mean free path (Fig. 6) in the energy range from 50 to $100 \mathrm{eV}$ is larger than the EMFP by factors 3 to 10 .

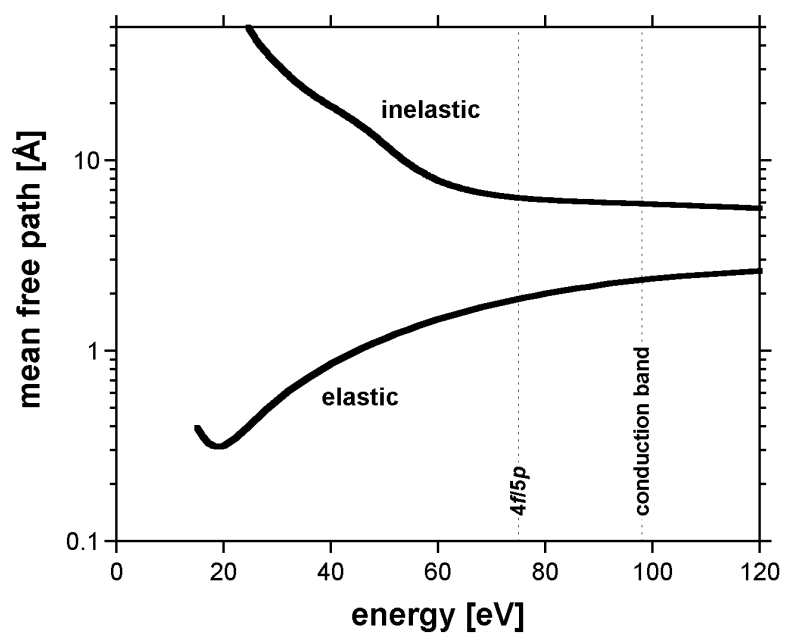

FIG. 6: Calculated elastic and inelastic mean free paths of electrons in tungsten. The energy is measured from the bottom of the conduction band $\left(E_{F}+W \approx 15 \mathrm{eV}\right)$.

Due to the smaller EMFP the average escape depth for electrons emitted without having suffered energy losses will be smaller than the IMFP. This enhances the surface sensitivity 
of the photoemission process. The total mean free path is determined by

$$
\frac{1}{\lambda_{t o t}}=\frac{1}{\lambda_{e l}}+\frac{1}{\lambda_{i n e l}}
$$

with $\lambda_{\text {tot }} / \lambda_{\text {inel,el }}$ being the probability for an (in-)elastic scattering process to happen. Between subsequent scattering processes electrons propagate in the time-varying electric field of the NIR laser. Travelling time, energy, and direction of motion are constantly updated. If an electron reaches the surface of the target it has to overcome the surface barrier which leads to an additional deflection at the surface. Energy lost in an inelastic scattering event is transferred to a secondary electron originating at the position of the collision. Its starting time is given by the time elapsed between start of the primary electron and the scattering event. As soon as the energy of an electron drops to below $25 \mathrm{eV}$ the trajectory calculation is stopped.

The effective mass of the energetic electron propagating through the crystal or, equivalently, its group velocity $v_{g}$ as determined from the dispersion relation $E(\vec{k})$, may have an important influence on the time dependence of the photoemission. To investigate the possible influence of the dispersion relation on the observed delay of emitted electrons we consider two limiting cases: a) a free-particle dispersion relation, $E=k^{2} / 2$, with effective mass $m_{\text {eff }}=1$ a.u. (red line in Fig. 7) and b) the distribution of group velocities (green line in Fig. 7) along the $\langle 110\rangle$ direction of W calculated by Silkin et al. (supplementary material to [8]). The latter features dips in the group velocity distribution around $65 \mathrm{eV}$ and $120 \mathrm{eV}$ with respect to the bottom of the conduction band due to the crystal potential. At both the expected energies of the core electrons and the conduction band (when measured from the bottom of the conduction band) we find $v_{g}$ very close to that of a free electron (Fig. (7). In Ref. [8], however, it was assumed that the energy of the core electrons would coincide with the local minimum of $v_{g}$ near $65 \mathrm{eV}$ (see Fig. 7). Under this assumption larger run time differences between core electrons and conduction band electrons would result. For test purposes and in order to maximize the effect of the crystal dispersion relation we followed this prescription and shifted the envelope of group velocities to match its local minimum at $65 \mathrm{eV}$ with the energy of the core electrons near $73 \mathrm{eV}$. The largest run-time differences reported in Sec. III were found under these somewhat arbitrary assumptions.

Finally, electrons escaping the surface are subject to the streaking field of the NIR laser 


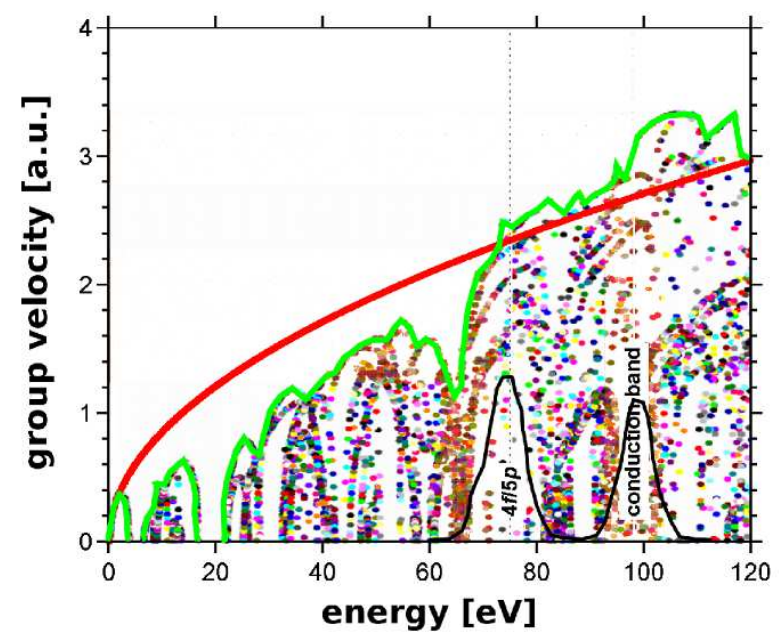

FIG. 7: (Color online) Group velocity distributions used in simulation; $v_{g}=k\left(m_{e f f}=1\right)$ (red line), envelope of calculated group velocities (green line) from the dispersion relation of $\mathrm{W}$ along the $\langle 110\rangle$ direction. Energies are measured with respect to the bottom of the conduction band; $E_{F}+W \approx 15 \mathrm{eV}$ with Fermienergy $E_{F}$ and workfunction $W$.

pulse. The latter transfers a momentum of

$$
\Delta p=\int_{t_{e x c}}^{t_{e n d}} E(t) d t
$$

to the electron where the integral is taken from the time of excitation to the conclusion of the laser pulse. Electrons with a final momentum oriented perpendicular to the surface with an acceptance angle $\Delta \theta= \pm 5^{\circ}$ about the surface normal are included in the streaking image. Parameters describing the NIR pulse (maximum field strength, wavelength, pulse duration) were taken from [8].

\section{RESULTS}

\section{A. Emission energy and emission time}

We first present results for energy and emission time distributions in the absence of the streaking field. Energy and emission time spectra are strongly affected by energy loss properties during transport to the surface. Fig. 8 depicts the two-dimensional correlated primary excitation - final escape energy distribution. In the absence of inelastic processes the electron distribution would be located on the diagonal. Due to collisions described by 


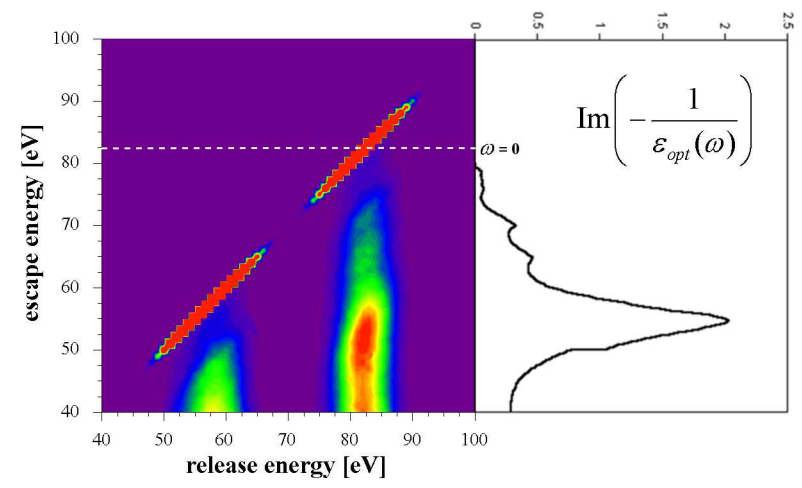

FIG. 8: (Color online) Primary excitation energy vs. escape energy for photoemission by an XUV pulse $(\hbar \omega=91 \mathrm{eV})$ from a $\mathrm{W}$ surface. The loss function of tungsten (right-hand side) shows a pronounced excitation (loss channel) at about $25 \mathrm{eV}$.

the energy loss function with a pronounced peak at about $25 \mathrm{eV}$, a significant portion of the initial distribution escapes at lower energy. Accidentally, the energy loss peak closely matches the energy spacing between core and conduction band electrons. Therefore, the primary conduction band electrons having undergone a single inelastic collision overlap with the primary energy distribution of the core electrons. The average excitation depth and traveling time in the target material for such electrons can be larger and will therefore contribute to the observed time delay. Also the secondary-electron background in the escape spectrum will be much larger in the spectral region of the core electrons adding to the observed run-time difference.

The two-dimensional correlated escape-time escape-energy distribution (Fig. 9) after the excitation by the XUV pulse feature consequently a much broader and slightly shifted escape time distribution of the "core" electron peak near $58 \mathrm{eV}$ compared to the conduction-band distribution near $83 \mathrm{eV}$. Note that the $t_{e s c}=0$ line corresponds to the temporal maximum of the XUV amplitude. The maxima of the distributions at 58 and $83 \mathrm{eV}$ are located close to 115 and 100 as, respectively. Averaging over the entire peak areas (44-70 eV, 70-95 eV) results in run times of 157 as and 115 as (see horizontal lines in Fig. 10). The run-time difference $(\sim 42$ as) can be accounted for in part by a simple estimate using the material properties of $\mathrm{W}$ : the average escape depth of electrons is given by their IMFP (see Fig. 6). Using the free-electron dispersion relation the total travel time of electrons with energies of 73 and $98 \mathrm{eV}$ (measured from bottom of conduction band) along their respective IMFP 


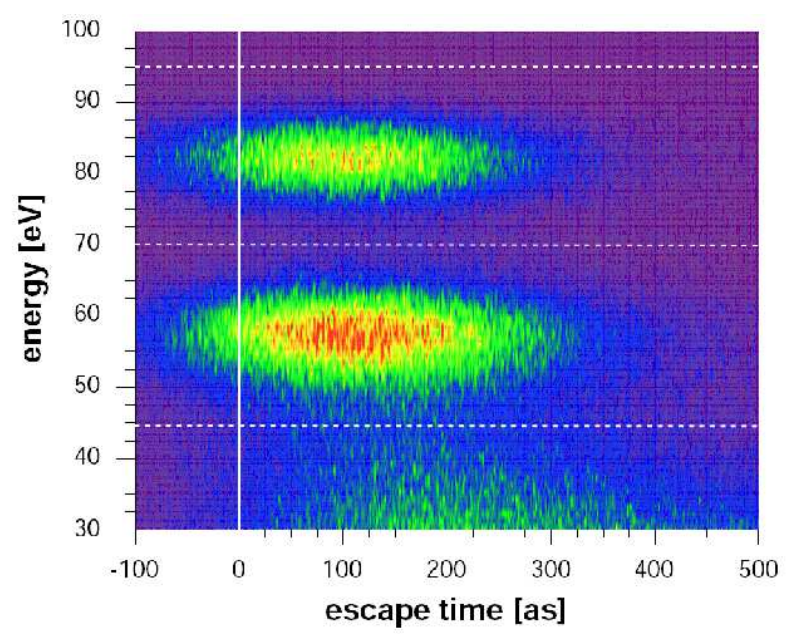

FIG. 9: (Color online) Correlated escape-time escape-energy distribution after excitation of a W surface by an XUV pulse $(\hbar \omega=91 \mathrm{eV}) \cdot t_{e s c}=0$ corresponds to the peak amplitude of the XUV pulse.

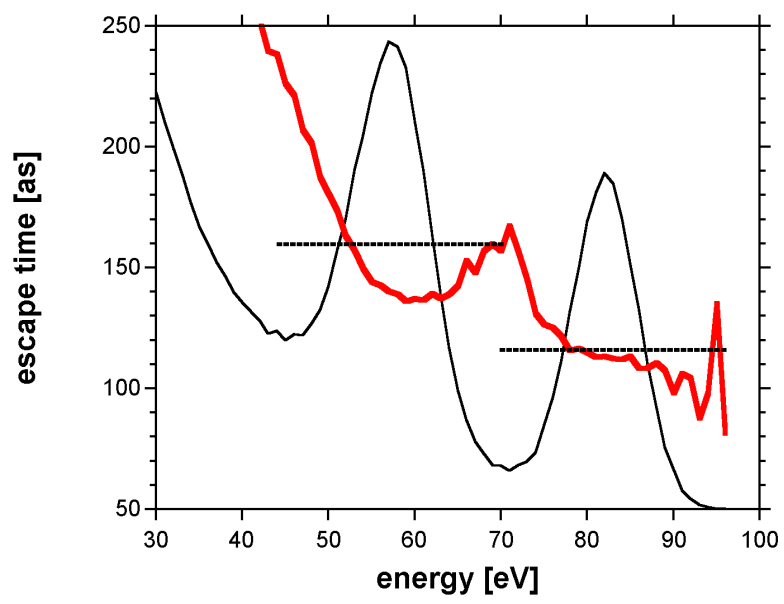

FIG. 10: (Color online) Energy spectrum (thin line) and escape times of electrons (thick red line) excited by an ultrashort XUV pulse. Horizontal lines indicate the escape time averaged over the core and conduction band peak regions. The mean run-time difference is about 40 as.

of $6.5 \AA$ and $5.8 \AA$ is about 125 as and 100 as, respectively. This gives a lower bound of the run-time difference for electrons with escape energies of $58 \mathrm{eV}$ and $83 \mathrm{eV}$ of about 25 as. This runtime difference is increased by scattering events in the target material which primarily increase the average run time of electrons ending in the spectral region of the core peak.

Projection onto the energy axis provides the spectrum while temporal information (Fig. 
10) is extracted by averaging the escape time over the electrons within a given escape energy bin. While at the maximum of the energy spektrum the run-time difference is only about 20 as, averaging over the entire energy range of the core and conduction band peaks (horizontal lines) results in a difference of 42 as. This is at the lower bound of the experimental value of $110 \pm 70$ as. The agreement would be considerably better when using the shifted envelope of group velocities as discussed above. In this case the run-time difference would be more than double and would increase to 101 as.

\section{B. Streaking images and center-of-mass motion}

The time structure of the photoelectron emission is extracted in the experiment [8] by attosecond streaking, i.e. by time-to-energy mapping in a few cycle NIR pulse. As soon as photoelectrons are excited they are subject to the NIR streaking field. If a free electron interacts with the entire laser pulse its momentum remains unchanged. If, however, it is set free while an electric field amplitude $E(t)$ is present at the position of excitation, a net momentum is transfered (Eq. 8). Varying the delay time between XUV pump and NIR probe pulses result in an oscillation of the final electron energy [7]. In the experiment streaking images have been recorded in delay-time steps of $\Delta \tau=100$ as. $\tau<0$ imply the probe pulse to precede the pump pulse, positive $\tau$ signify the pump pulse preceding the probe.

The characteristic streaking oscillations in the simulated electron energy can be clearly seen (Fig. 11). From the streaking image a mean run-time difference of 33 as between core electrons (integrated over the energy interval $44 \leq E \leq 70 \mathrm{eV}$ ) and the conduction electrons (70 $\leq E \leq 95 \mathrm{eV}$ ) can be deduced. The values deduced from the streaking image is slightly lower (by about 10 as) than that directly deduced from the simulated time spectrum. This discrepancy is not due to the resolution limits of the streaking technique but consequence of the modification of the electron transport by the streaking field. Electron trajectories are deflected in the direction of the NIR laser polarization inside the target. As a consequence, the escape depth of excited electrons is reduced. The point to be emphasized is that the streaking field not only probes but actively modifies the emission time spectrum. Along the same lines, the streaking field also alters the final emission energy spectrum (Fig. 12). Taking the streaking field into account, the shape of the electron spectra drastically changes. Almost 


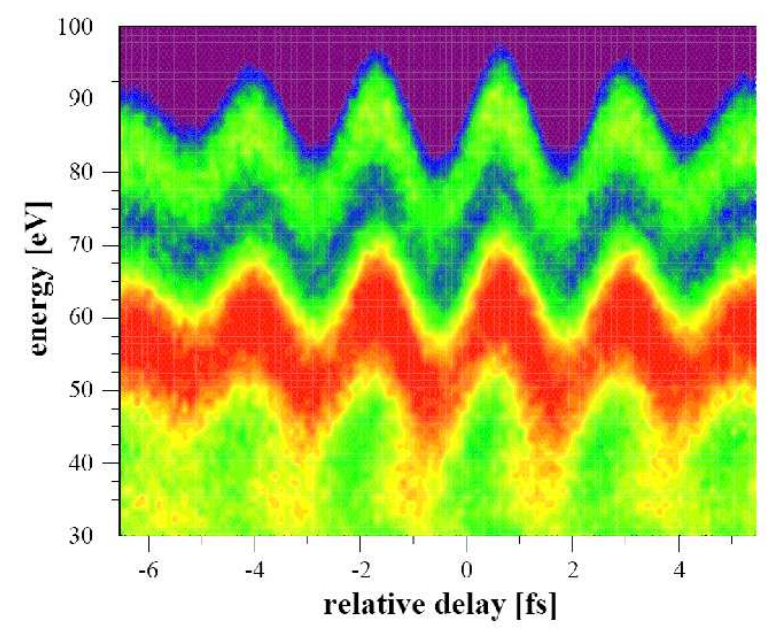

FIG. 11: (Color online) Calculated streaking images for electrons emitted by a 300 as XUV pulse and streaked by a few-cycle NIR pulse. Emission along the surface normal with an acceptance cone angle of $\theta=0 \pm 5^{\circ}$.

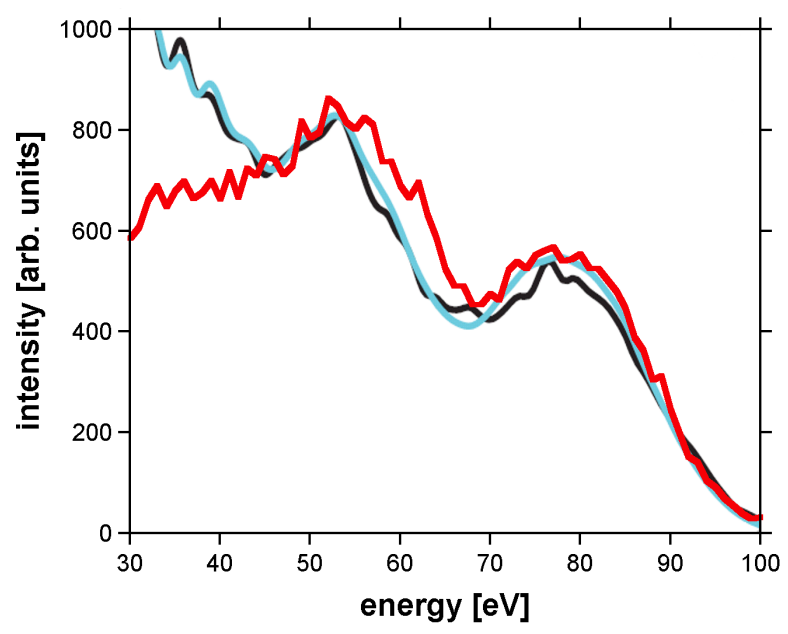

FIG. 12: (Color online) XUV pulse induced photoemission spectrum in the presence of a NIR streaking field. Thick red line: simulated spectrum for electrons emitted in the direction of the surface normal with an acceptance cone of half-width $\Delta \theta=5^{\circ}$; black line: experiment; blue line: smoothed experimental data.

perfect agreement between simulation and experiment is achieved. Both the simulation and the experiment feature broadened core and conduction band peaks (compare Figs. 5 and 12). The overall agreement in peak height and width indicates that the present simulation is capable of accounting for most of the relevant processes governing the XUV-pulse induced 
photoemission from a tungsten surface.

\section{CONCLUSION}

We have presented simulations of the electron excitation, transport, and attosecond streaking for an XUV-pump-NIR-probe setting near a tungsten surface. This scenario models the recent experiment by Cavalieri et al. [8]. We find excellent agreement for the energy spectrum in the presence of the streaking field. In agreement with the experiment we find the core electron emission to be delayed relative to the conduction band electrons. Calculated run-time differences between groups of photoelectrons are at the lower bound of the error bar of the measurement. Latest experiments indicate a somewhat smaller run-time difference of $85 \pm 45$ as [20] reducing the gap between measured data and our simulations. Processes responsible for the time delay identified by the present simulation include the larger emission depth of core electrons, the contribution of primary emitted conduction electrons slowed down to energies matching emitted core electrons as well as secondary electrons. When we include a modification of the group velocity distribution for energies in the region of core electrons as proposed by Silkin et al. [8], the delay time increases and is closer to the experimental data. We note, however, that invoking this correction would require an energy shift of the spectrum for which a convincing explanation is missing. Possible other mechanisms not yet accounted for include the influence of the NIR pulse on the primary photoexcitation process and local crystal field effects on the emission time spectrum. The impact of both effects could be increased by local field enhancements at the surface (surface

plasmons) as recently shown for the nanoplasmonic field microscope [27]. Such fields might influence excitation and transport of photoelectrons from the conduction band and bound states differently.

\section{Acknowledgments}

This work was supported by the Austrian Fonds zur Förderung der wissenschaftlichen Forschung under grants no. FWF-SFB016 "ADLIS" and no. 17449, the European ITSLEIF network RII3\#026015, and the TeT Grant No. AT-7/2007. One of us (KT) was also partially supported by the grant "Bolyai" from the Hungarian Academy of Sciences 
and the Hungarian National Office for Research and Technology. We thank the group of R. Kienberger (MPQ Garching) for making their latest data available to us prior to publication.

[1] D.A. Shirley, Phys. Rev. B 5, 4709 (1972).

[2] W. Friedrich, P. Knipping, and M. von Laue, Sitzungsberichte der MathematischPhysikalischen Classe der Königlich-Bayerischen Akademie der Wissenschaften zu München 1912: 303 (1912).

[3] K. Siegbahn, Rev. Mod. Phys. 54, 709 (1982).

[4] T. Ohta, A. Bostwick, J.L. McChesney, T. Seyller, K. Horn, and E. Rotenberg, Phys. Rev. Lett. 98, 206802 (2007).

[5] E.A. Stern, Phys. Rev. B 10, 3027 (1974).

[6] M. Echenique and J.B. Pendry, J. Phys. C 11, 2065 (1978).

[7] R. Kienberger, E. Goulielmakis, M. Uiberacker, A. Baltuska, V. Yakovlev, F. Bammer, A. Scrinzi, Th. Westerwalbesloh, U. Kleineberg, U. Heinzmann, M. Drescher, and F. Krausz, Nature 427, 817 (2004).

[8] A.L. Cavalieri, N. Müller, Th. Uphues, V.S. Yakovlev, A. Baltuska, B. Horvath, B. Schmidt, L. Blümel, R. Holzwarth, S. Hendel, M. Drescher, U. Kleineberg, P.M. Echenique, R. Kienberger, F. Krausz, and U. Heinzmann, Nature 449, 1029 (2007).

[9] J. Burgdörfer and J. Gibbons, Phys. Rev. A 42, 1206 (1990).

[10] Handbook of Optical Constants of Solids, ed. E.D. Palik (Academic Press, San Diego, 1985).

[11] A.G. Eguiluz, Phys. Rev. B 23, 1542 (1981).

[12] H.B. Rose, A. Fanelsa, T. Kinoshita, Ch. Roth, F.U. Hillebrecht, and E. Kisker, Phys. Rev. B 53, 1630 (1996).

[13] J.-J. Yeh and I. Lindau, At. Data Nucl. Data Tables 32, 1 (1985).

[14] E. Christensen and B. Feuerbacher, Phys. Rev. B 10, 2349 (1974).

[15] R.W. Strayer, W. Mackie, L.W. Swanson, Surf. Sci. 34, 225 (1973).

[16] L.F. Mattheiss, Phys. Rev. 139, A1893 (1965).

[17] X. Gonze, J.M. Beuken, R. Caracas, F. Detraux, M. Fuchs, G.M. Rignanese, L. Sindic, M. Verstraete, G. Zerah, F. Jollet, M. Torrent, A. Roy, M. Mikami, Ph. Ghosez, J.-Y. Raty, and D.C. Allanet, Comp. Mat. Science 25, 478 (2002). 
[18] A.M. Rappe, K.M. Rabe, E. Kaxiras, and J.D. Joannopoulos, Phys. Rev. B 41, 1227(R) (1990); Erratum Phys. Rev. B 44, 13175 (1991).

[19] XPS spectra at http://www.lasurface.com.

[20] A.L. Cavalieri, R. Ernstorfer, and R. Kienberger, J. Phys. B, submitted (2009); private communication (2009).

[21] B. Solleder, C. Lemell, K. Tökesi, N. Hatcher, and J. Burgdörfer, Phys. Rev. B 76, 075115 (2007).

[22] F. Salvat, A. Jablonski, and C. Powell, Comp. Phys. Commun. 165, 157 (2005).

[23] C.O. Reinhold and J. Burgdr̈fer, Phys. Rev. A 55, 450 (1997).

[24] Z.J. Ding and R. Shimizu, Surf. Sci. 222, 313 (1989).

[25] C.J. Powell, Surf. Sci. 44, 29 (1974).

[26] D.R. Penn, Phys. Rev. B 35, 482 (1987).

[27] M.I. Stockman, M.F. Kling, U. Kleineberg, and F. Krausz, Nature Photonics 1, 539 (2007). 